\title{
Meditation promotes insightful problem-solving by keeping people in a mindful and alert conscious state
}

\author{
REN Jun ${ }^{1 *}$, HUANG ZhiHui ${ }^{1}$, LUO Jing ${ }^{2 *}$, WEI GaoXia ${ }^{2}$, YING XiaoPing ${ }^{3}$, DING ZhiGuang ${ }^{4}$, \\ WU YiBin ${ }^{5} \&$ LUO Fei ${ }^{2}$ \\ ${ }^{1}$ College of Education, Zhejiang Normal University, Jinhua 321004, China; \\ ${ }^{2}$ Key Laboratory of Mental Health, Chinese Academy of Sciences, Beijing 100101, China; \\ ${ }^{3}$ Institute of Sociology, Chinese Academy of Social Sciences, Beijing 100026, China; \\ ${ }^{4}$ Department of Social Sciences, Hebei Medical University, Shijiazhuang 050015, China; \\ ${ }^{5}$ Beijing Easy Monitor Technology Co., Ltd., Beijing 100101, China
}

Received December 23, 2010; accepted June 27, 2011

\begin{abstract}
Although previous studies have shown that sleep can inspire insight, it is still unclear whether meditation can promote insight. Meditation differs from other types of passive rest such as relaxation and sleep because it requires full consciousness and mindfulness of targets such as one's breathing. Forty-eight university students without meditation experience were recruited to learn a simple meditation technique. They were given a list of 10 insight problems to solve (the pre-test session). In this study, we focused on the unsolved problems and examined if they could be successfully solved after a 20 min rest interval with or without meditation. Results showed that relative to the control group that listened to Chinese or English words and made a language judgment, the groups who learned meditation successfully solved significantly more failed problems from the pre-test session, providing direct evidence for the role of meditation in promoting insight. Further analysis showed that maintaining a mindful and alert state during meditation (raising a hand to report every 10 deep breaths compared to every 100 deep breaths) resulted in more insight regarding the failed items from the pre-test session. This implies that it was watchfulness in meditation, rather than relaxation, that actually contributed to insight. Consistently, in the meditation session or control task, the percentage of alpha waves - a brain index of mental relaxation — was negatively correlated with insight. These results suggest a meditation-based insight-promoting mechanism different from that involved in passive rest such as relaxation and sleep.
\end{abstract}

meditation, insight, problem solving

Citation: Ren J, Huang Z H, Luo J, et al. Meditation promotes insightful problem-solving by keeping people in a mindful and alert conscious state. Sci China Life Sci, 2011, 54: 961-965, doi: 10.1007/s11427-011-4233-3

It has been shown that sleep can significantly increase insightful awareness, for example, discerning a hidden principle needed to solve the Number Reduction Task (NRT) [1]. However, it is still unclear whether meditation can promote insight. This question deserves to be explored because meditation differs from routine forms of rest such as sleep and relaxation in that one's attention is actively focused on the present state of consciousness [2-7]. It is widely believed in

*Corresponding author (email: renj@zjnu.cn; luoj@psycy.ac.cn)
Asian cultural traditions such as Buddhism and Taoism that meditation can lead to insight.

This study recruited undergraduate students without any previous meditation practice as participants. They were taught a simple method of meditation and were then tested to determine if application of meditation could promote insight. Different from Wagner and colleagues' experiment that adopted the NRT [1], we used 10 typical insight problems [8]. We first instructed participants to try to solve these problems one by one. We selected the unsolved problems 
for each participant for later testing. Then, we required participants to engage in either $20 \mathrm{~min}$ of meditation or a control cognitive task. Finally, participants were unexpectedly re-administered their unsolved problems from the first session and asked to re-think and solve these problems. The key hypothesis of this study was that participants who had learned meditation would be more capable of insightfully solving the unsolved problems.

To specify how meditation promotes insight, the effect of meditation with different levels of alertness and awareness was examined. These levels were manipulated through changing the frequency of self-reported (by hand-raising) number of deep breaths. During meditation, participants were required to clear their minds, focus on deep breathing, and count number of breaths. In the M10 condition, participants were required to raise their hand to report every 10 breaths. In the M100 condition, participants reported every 100 breaths by raising their hand. Both M10 and M100 met the criterion of meditation, but relative to M100, M10 participants stayed in a more alert and awake state because they were required to frequently report their number of breaths.

During the meditation and control cognitive task period, electroencephalograms of participants were recorded to monitor and evaluate mind-brain states. Some EEG indices, such as percentage of alpha waves, which indicate level of mental relaxation, as well as the score of i22 related to attention focus [9], were considered in obtaining crossdomain evidence for the efficiency of our experimental manipulation and for suggesting possible mental mechanisms related to how meditation promotes insight.

\section{Materials and methods}

\subsection{Participants}

Forty-eight healthy undergraduate students (23 males, mean age 23.3 years) recruited from a university in Beijing participated in the study. They were randomly assigned to three equal groups with balanced male/female ratios: meditation group M10 (reported every 10 deep breaths), meditation group M100 (reported every 100 deep breaths), and the control group. There were 16 participants in each group.

\subsection{Materials}

Fifteen insight problems that met the criterion of cognitive reconstruction were used [8]. Five were used as examples in the instruction and practice session, and 10 were used for the formal experiment.

\subsection{EEG recording and analysis}

EEG data were collected by HXD-I EEG equipment (HUAXIANG Technology Company, Harbin, Heilongjiang, China). The collecting electrodes were located in the bilat- eral prefrontal cortex, reference electrodes were A1 and A2, and leading electrodes were located in the center of frontal sites. The frequency was $200 \mathrm{~Hz}$, the time window for analysis was 1.25 , the arithmetic stacking coefficient was 6 , the input impedance was $9 \Omega$, the resolution of EEG was 3 $\mathrm{MV}$, and the frequency band was $0.5-100 \mathrm{~Hz}$. Wavelet analysis, spectrum analysis, and pattern recognition were applied in EEG analysis to extract the wavelet index. The main indices included alpha waves, i-35 related to relaxation and i-22 related to alertness. The latter two indices were developed based on our clinical and experimental trials and can be used as a reference index for common EEG data [9]. The EEG data recording for the last 15 min was analyzed.

\subsection{Procedure}

\subsubsection{Meditation and problem-solving training}

Before the formal experimental session, participants were taught Susoku meditation, which requires participants to mindfully control and focus on their deep abdominal breathing [10-12]. In our pilot study, we found a cartoon computer animation very helpful for beginners learning the skill of deep abdominal breathing. Participants were asked to attentively and slowly take a breath in or out as the "inspiration fish" or "expiration fish" gradually and smoothly went up or down on the computer screen. Each round of inspiration and expiration lasted $7 \mathrm{~s}$. After participants grasped the method of deep abdominal breathing, they were further instructed to attentively take inspiration and expiration breaths without the external aid of the computer animation (with eyes slightly closed). They were also instructed to do their best to be mindfully aware and disengage from any irrelevant thinking, free association, or feelings that might incidentally occur. During meditation, participants were asked to count the number of their breaths from 1 to 10 and then restart (the M10 condition), or from 1 to 100 and then restart (the M100 condition). They were also asked to raise their hand to report finishing one round of breaths (i.e., 10 breaths in M10 or 100 breaths in M100). The abovementioned training procedures as well as subsequent problem solving and meditation practice were conducted with participants individually. Participants in the control group did not learn meditation. They rested in the waiting room for the same period of time.

After meditation (the M10 and M100 group) or rest (the control group), all three groups were given five examples of insight problems for practice. Every problem was presented on the screen for a maximum of $3 \mathrm{~min}$. If participants thought the problem was successfully solved, they were instructed to press the " 2 " button as soon as possible and then write down the solution. If participants could not solve the problem, they could wait until the problem disappeared from the screen at the end of the 3 min maximum, or press the " 3 " key to stop the presentation and go to the next item. 
It was emphasized that participants should not continue to think about the unsolved problem once it disappeared from the screen. Participants did not know they would be given the unsolved problems later and would have to try to solve them again.

\subsubsection{Formal experimental session}

The experimental session included the pre-test, the interval, and the post-test.

(i) Pre-test. In the pre-test phase, participants were instructed to solve 10 insight problems appearing randomly one by one on the screen using the same procedure as in the practice session.

(ii) Meditation or control cognitive task interval. During this interval, the M10 and M100 groups were instructed to count number of breaths for 20 min, while the control group was instructed to complete an auditory judgment task comprising English and Chinese words. The ratio of Chinese words to English words was 1 to 6 . Words appeared randomly with an ITI of $5 \mathrm{~s}$. Participants were instructed to raise their hand when they heard the Chinese words and not make any response when they heard the English words. EEGs were recorded during meditation and the control cognitive task. In view of the possible unstable state of participants, the EEG recording for the last 15 min was analyzed.

(iii) Post-test. After the meditation or control cognitive task interval, participants were given the problems they had failed in the pre-test session and were required to try to solve these problems again. The procedure was the same as in the pre-test session.

\section{Results}

\subsection{Behavioral results}

\subsubsection{Successful problem-solving rate}

Problem-solving rate included problem-solving rates in the pre- and post-tests. It was defined as the number of successfully solved items in the pre- or post-test divided by the total number of items (total number of items in the post-test was the 10 total items minus successfully solved items in the pre-test). This step aimed to compare differences in problem solving ability among these three groups. An ANOVA showed that there was no significant difference among the three groups in problem-solving rate for the pre-test $(P>0.05$, Table 1$)$, revealing that participants in the

Table 1 Problem-solving rate in the pre-test ${ }^{\mathrm{a})}$

\begin{tabular}{ccccc}
\hline Group & Number of participants & M & SD & SE \\
\hline M10 & 16 & 0.49 & 0.15 & 0.04 \\
M100 & 16 & 0.54 & 0.15 & 0.04 \\
Ctrl & 16 & 0.56 & 0.14 & 0.03 \\
Total & 48 & 0.53 & 0.15 & 0.02 \\
\hline
\end{tabular}

a) The M10 group reported every 10 breaths, the M100 group reported every 100 breaths, and Ctrl represents the control group. three groups did not differ in their problem-solving ability.

Our study was mainly concerned with the problemsolving rate in the post-test, which would suggest whether the intervention (meditation) exerted an effect on problem solving. Nonparametric statistics (Kruskal-Wallis $H$ test) were used because the data for problem-solving rate in the post-test were abnormally distributed. The results showed a significant difference among problem-solving rates in the post-test $(\chi$-square $=11.414, \mathrm{~d} f=2, P<0.005)$. Further analysis with the Mann-Whitney $U$ test showed that the problem-solving rate of the M10 group was higher than that of the M100 group $(P=0.051)$ and significantly higher than that of the control group $(P<0.005)$. The problem-solving rate of the M100 group was higher than that of the control group $(P<0.05$, Table 2$)$. These results revealed that (i) problem-solving ability of the two meditation groups was better than that of the control group, and (ii) the performance of the M10 group was better than that of the M100 group.

\subsubsection{Response times (RTs)}

First, we calculated the average response time (RT) for problem solving (including successful and unsuccessful tries) in the three conditions. Results showed that in the pre-test, the RTs in the three conditions were not significantly different and there was no interaction effect $(P=0.381)$. Using RTs in the pre-test as covariates in the analysis, we found no significant difference or interaction effect among the three groups at post-test $(P=0.792$, Table 3).

Then, we compared RTs for successfully solved problems and abandoned (failed) problems among the three conditions. Results showed that the three groups did not differ in RTs and showed no interaction effect in the pre-test for both kinds of problems $(P=0.325$ for solved problems, $P=0.830$ for abandoned problems). With RTs for successfully solved problems or abandoned (failed) problems in the pre-test as covariates, the analysis revealed no significant difference or interaction effect at post-test $(P=0.378$ for successfully solved problems, $P=0.945$ for abandoned problems; Tables 4 and 5) among the three conditions.

\subsection{EEG results}

In this study, we examined the percentage of alpha waves in

Table 2 Problem-solving rate in the post-test ${ }^{\mathrm{a})}$

\begin{tabular}{cccc}
\hline & \multicolumn{3}{c}{ Group comparisons } \\
\cline { 2 - 4 } & M10-M100 & M10-Ctrl & M100-Ctrl \\
\hline Mann-Whitney U & 77.50 & 50.00 & 70.50 \\
Wilcoxon W & 213.50 & 186.00 & 206.50 \\
Z & -1.92 & -3.01 & -2.23 \\
\hline
\end{tabular}

a) The M10 group reported every 10 breaths, the M100 group reported every 100 breaths, and Ctrl represents the control group. 
Table 3 RTs in the pre-test and post-test

\begin{tabular}{ccccc}
\hline & Group & Number of participants & M (ms) & SD \\
\hline Pre-test & M10 & 16 & 71185.43 & 16873.03 \\
& M100 & 16 & 76823.79 & 17143.16 \\
& Ctrl & 16 & 81040.91 & 24739.61 \\
& Total & 48 & 76350.04 & 19915.76 \\
Post-test & M10 & 16 & 99166.95 & 34191.55 \\
& M100 & 16 & 101111.43 & 33481.85 \\
& Ctrl & 16 & 96973.40 & 40199.79 \\
& Total & 48 & 99083.93 & 35349.00 \\
\hline
\end{tabular}

Table 4 RTs of successfully solved problems in the pre-test and post-test ${ }^{\mathrm{a}}$

\begin{tabular}{ccccc}
\hline & Group & Number of participants & M (ms) & SD \\
\hline Pre-test & M10 & 16 & 56369.91 & 18393.58 \\
& M100 & 16 & 61182.66 & 15213.48 \\
& Ctrl & 16 & 67644.36 & 31260.17 \\
& Total & 48 & 61732.31 & 22704.73 \\
Post-test & M10 & 15 & 78005.15 & 36924.43 \\
& M100 & 15 & 67062.57 & 41478.93 \\
& Ctrl & 6 & 59237.92 & 38748.66 \\
& Total & 36 & 70317.87 & 38716.58 \\
\hline
\end{tabular}

a) Participants who did not solve any problems correctly were eliminated from the post-test and were regarded as missing values.

Table 5 RTs of the abandoned (failed) problems ${ }^{\text {a) }}$

\begin{tabular}{ccccc}
\hline & Group & Number of participants & M (ms) & SD \\
\hline Pre-test & M10 & 16 & 90526.28 & 24606.78 \\
& M100 & 13 & 94610.68 & 25597.84 \\
& Ctrl & 13 & 96884.51 & 34869.44 \\
& Total & 42 & 93758.52 & 27867.09 \\
Post-test & M10 & 9 & 102690.22 & 41354.98 \\
& M100 & 10 & 104566.24 & 44002.92 \\
& Ctrl & 13 & 109053.26 & 43440.90 \\
& Total & 32 & 105861.46 & 41733.89 \\
\hline
\end{tabular}

a) Participants who solved all problems correctly were eliminated from the pre- and post-tests and were regarded as missing values.

the three conditions. A higher percentage of alpha waves have been associated with a more relaxed mental state. A one-way within-groups ANOVA showed a significant difference in the percentage of alpha waves among the three groups $(F(2,45)=4.144, P<0.05)$. Post-hoc testing showed that the percentage of alpha waves in the M10 group was significantly lower than in the control group $(P<0.05)$ and showed a decrease in tendency relative to that of the M100 group $(P=0.098)$. There was no difference in the percentage of alpha waves between the M10 group and control group (Table 6). This indicated that the M10 group stayed more alert during the meditation interval session. Productmoment correlation analysis across all participants in the three groups revealed that percentage of alpha waves was negatively correlated with problem-solving rate in the post-test $(r=-0.471, P=0.001)$.

We also analyzed i-35 and i-22, which are shown to be related to states of attention and general relaxation, respec- tively. Results showed there was a significant difference in i-35 among the three groups $(F(2,45)=6.516, P<0.005)$. Post-hoc testing revealed that the M10 group had higher i-35 than the M100 group $(P<0.05)$ and control group $(P=0.073)$, but no significant difference existed between the M100 group and the control group. This again indicated that the latter two groups were more relaxed than the M10 group. As for i-22, the results showed differences among the three groups $(F(2,45)=3.377, P<0.05)$. Post-hoc testing showed that the M10 group showed a lower i-22 tendency than the M100 group $(P=0.084)$ and control group $(P=0.093)$. However, there was no difference between the M100 group and control group. This implied that participants in the M10 group were more mentally focused than the M100 and control group. The correlation analysis showed that i-35 was not correlated with problem-solving rate in the post-test, and i-22 was negatively correlated with problem-solving rate in the post-test $(r=-0.403, P<0.005)$. These results indicated that a more alert and focused mind in the meditation or control cognitive task interval may have been associated with better problem-solving performance in the post-test.

\section{Discussion}

Our results showed that after 20 min of meditation or a control cognitive task, participants were able to solve some of the problems they had failed in the pre-test. This is generally in line with previous research on the role of incubation. However, participants who engaged in meditation solved more previously unsolved problems compared to participants in the control condition, thereby providing direct evidence for the role of meditation in promoting insight.

The following considerations excluded, or partially excluded, the possibility that the observed differences between M10, M100, and Ctrl conditions were caused by other unrelated factors. First, in the initial problem solving session (pre-test), there were no significant differences in solution rate and RTs among the three groups, thus excluding the possibility that the observed key differences were caused by differences in problem solving ability in the groups. Second, in the final problem solving session (post-test) after meditation or the control cognitive task, there was no detectable difference in RTs for problem solving among the three groups, thus excluding the possibility that the observed differences were caused by participants in the M10 and M100 groups having learned to be more patient and insistent on solving the problems after meditation. Further, we also calculated the RTs for the successfully solved items and failed items for the three groups. We found that in neither the initial session nor the final session was there any detectable difference among the groups. This excluded the possibility that the better performance of the M10 and M100 groups was related to the increase of participants' meta-cognitive ability in identifying the solvable and unsolvable items, thus ena- 
bling them to wisely spend more time on the solvable items.

Rather, it is reasonable to propose that the increased insight ability brought about by meditation may be related to the fact that the participants had maintained a more alert and mindful state of consciousness. First of all, participants in M10, relative to M100, showed a stronger tendency for insight. Although the difference was not significant, it approached significance $(P=0.051)$. Secondly, there was a significant negative correlation between insightful problem solving in the final session and the percentage of alpha waves known to be closely related to mental relaxation. This implies that the more relaxed the participants were in the meditation or control cognitive task interval, the less insight they achieved later. Thirdly, the percentage of alpha waves in M10 was not only significantly lower than in the Ctrl condition, but also lower than that in M100 (but did not reach significance, $P=0.098$ ). Taken together, these findings imply that meditation promoted insight by keeping people in an alert and mindful state. This appears to be different from the possible mechanism underlying promotion of insight during a passive type of relaxation, like sleep, that does not require any mental effort.

This work was supported by the Education Program of the National Social Science Foundation of China (Grant No. BBA100016), the National Basic Research Program of China (Grant No. 2010CB833904), the National Natural Science Foundation of China (Grant Nos. 30970890 and 30770708), and the National High Technology Research and Development Program of China (Grant No. 2008AA022604).
1 Wagner U, Gais S, Haider H, et al. Sleep inspires insight. Nature, 2004, 427: 352-355

2 Tang Y Y, Lu Q, Geng X, et al. Short-term meditation induces white matter changes in the anterior cingulate. Proc Natl Acad Sci USA, 2010, 107: 15649-15652

3 Tang Y Y, Ma Y, Fan Y, et al. Central and autonomic nervous system interaction is altered by short-term meditation. Proc Natl Acad Sci USA, 2009, 106: 8865-8870

4 Tang Y Y, Ma Y, Wang J, et al. Short-term meditation training improves attention and self-regulation. Proc Natl Acad Sci USA, 2007, 104: 17152-17156

5 Slagter H A, Lutz A, Greischar L L, et al. Mental training affects distribution of limited brain resources. PLoS Biol, 2007, 5: e138

6 Lutz A, Slagter H A, Rawlings N B, et al. Mental training enhances attentional stability: neural and behavioral evidence. J Neurosci, 2009, 29: 13418-13427

7 Lutz A, Slagter H A, Dunne J D, et al. Attention regulation and monitoring in meditation. Trends Cogn Sci, 2008, 12: 163-169

8 Weisberg R W. Prolegomena to theories of insight in problem solving: A taxonomy of problems. In: Sternberg R J, Davidson J E, eds. The Psychology of Problem Solving. Cambridge: Cambridge University Press, 1995. 157-196

9 Luo F, Luo J, Wu Y B, et al. Psychosomatic recovery by relaxing and rebuilding the brain: Possible application in post traumatic psychological recovery (in Chinese). Adv Psychol Sci, 2009, 17: 594601

10 Takahashi T, Murata T, Hamada T, et al. Changes in EEG and autonomic nervous activity during meditation and their association with personality traits. Int J Psychophysiol, 2005, 55: 199-207

11 Murata T, Takahashi T, Hamada T, et al. Individual trait anxiety levels characterizing the properties of Zen meditation. Neuropsychobiology, 2004, 50: 189-194

12 Kubota Y, Sato W, Toichi M, et al. Frontal midline theta rhythm is correlated with cardiac autonomic activities during the performance of an attention demanding meditation procedure. Brain Res Cogn Brain Res, 2001, 11: 281-287

Open Access This article is distributed under the terms of the Creative Commons Attribution License which permits any use, distribution, and reproduction in any medium, provided the original author(s) and source are credited. 